\title{
0 Design de Informação e a narrativa diagramática em Whale Hunt de Jonathan Harris
}

Juliana Rocha Franco

\section{Resumo:}

Uma questão que atravessou os anos 80 e 90 do século passado, a "hipertrofia das imagens" no mundo contemporâneo, poderia ser nos dias de hoje reformulada e ampliada. Vive-se atualmente uma imersão na hipertrofia dos dados na qual as modalidades de criação, organização e acesso à informação têm se alterado drasticamente. Dentro desse contexto, o uso de bancos de dados e ferramentas interativas que promovem o Design de informação tem se revelado importantes para práticas de produção de sentido e organização no mundo contemporâneo. $O$ presente trabalho busca compreender a potencialidade narrativa dos ambientes midiáticos de visualização dinâmicos de bancos de dados digitais. Especificamente é empreendido um estudo de caso da obra Whale Hunt, do artista multimídia Jonathan Harris. O que buscaremos mostrar no presente trabalho, é que uma interface pode ser um poderoso dispositivo narrativo ao ser trabalhada diagramaticamente. Em Whale Hunt o usuário não só? interpreta a narrativa, mas como também a explora e a configura. A premissa básica é a da agência como interação.

Palavras-chave: Visualização de Informação, Diagrama; Narrativa, Banco de dados.

\section{Abstract:}

Currently the modalities for the creation, organization and access to information have changed dramatically. Within this context, the use of databases and interactive information visualization tools brought new forms of creation and has proved to be an important element to production practices of meaning and organization in the contemporary world. The work seeks to understand the potential of narrative media environments of dynamic display of digital databases. Specifically, it will be undertaken a case study of the work Whale Hunt, by the multimedia artist Jonathan Harris. In Whale Hunt the user not only interprets the narrative, but also explores and 
configures it. The basic premise of this kind of narratives is the agency as interaction. Through theory of Charles S. Peirce's theory of diagrammatic reasoning work, we will seek to show that an interface can be a powerful narrative device, producing knowledge diagrammatically.

Keywords: Information visualization, Diagram; Narrative, Data set.

\title{
0 mundo imerso nos dados: visualização de informação e narrativa
}

Vários autores enfatizam o crescente aumento no volume de dados produzidos atualmente (HOU; PAI, 2009; MEIRELLES, 2013; TERGAN, 2005; VESNA, 2007). Segundo Meirelles (2007, p.45), a necessidade de organizar e visualizar grandes quantidades de dados e sistemas complexos tem aumentado nos últimos anos em todos os domínios. Quaggiotto ressalta que o conhecimento tem sido cada vez mais considerado em termos de acesso à informação:

\begin{abstract}
Knowledge is less and less considered in terms of possession, and increasingly in terms of access to information and skills. Knowing means having access to social and technological networks able to provide necessary information and insight at the time of need. The rigid and permanent structures of disciplines and subject areas are therefore progressively replaced by fluid and dynamic spaces in permanent movement and evolution, able to define cross-cutting paths, areas of interest and clusters (QUAGGIOTTO, 2010, p.1).
\end{abstract}

Bases de dados permeiam a vida moderna, e grande volume de dados necessitam de indexação e de organização. O conceito de Banco de dados já é amplamente conhecido relacionado com a organização de dados e informações. Segundo Guimarães (2003, p. 19), um banco de dados "uma coleção de dados ou informações relacionadas entre si. Elas representam aspectos do mundo real com significado próprio e que desejamos armazenar para uso futuro". No entanto, para além de uma abordagem meramente informática, o presente trabalho compreende o banco de dados como um artefato cultural e que segundo 
Manovich possibilita "uma nova forma de estruturar a nossa experiência de nós mesmos e do mundo" (p. 40). Atualmente, análises de grandes bancos de dados tem conduzido processos de tomada de decisão em organizações, mercado financeiro, publicidade, etc.

No contexto de hipertrofia dos dados, a ideia de visualização de informação é profundamente eficaz para o tratamento e gestão de conhecimento e informação (TERGAN, 2005, p.1). Conforme afirma Chen (2006, p. 1), a visualização de informação, como um campo distinto de pesquisa, é recente, mas, no entanto, tem se tornado um campo de pesquisa interdisciplinar de longo alcance. O autor também enfatiza que a fronteira entre a visualização da informação e áreas afins, tais como a visualização científica e modelagem de simulação está se tornando cada vez mais tênue. Referencias à visualização de informação são encontrados na literatura de um grande número de domínios disciplinares.

Manovich (2011, p. 37) afirma que apesar da crescente popularidade da visualização da informação, não é tão fácil apresentar uma definição que sirva para todos os tipos de projetos criados atualmente, e, ao mesmo tempo, que a separe claramente de campos correlatos, como por exemplo visualização científica e design de informação. O autor propõe uma definição na qual o que ele chama de design da informação lidaria com a informação e a visualização de informação lidaria com dados:

Information design starts with the data that already have a clear structure, and its goal is to express this structure visually. For example, the famous London tube map designed in 1931 by Harry Beck uses structured data: tube lines, tube stations and their locations over London geography. In contrast, the goal of information visualization is to discover the structure of a (typically large) data set. This structure is not known a priori; visualization is successful if it reveals this structure. (MANOVICH, Lev, 2011, p.38) 
Ou seja, o design da informação lida com dados que já possuem uma estrutura clara e sua meta é a de expressar essa estrutura visualmente (MANOVICH, Lev, 2011, p.38) e a visualização de informação envolve representações visuais de dados abstratos. Ao fim e ao cabo, ambos revelam visualmente padrões e relações que não seriam facilmente deduzidos sem representações gráficas. Sua distinção básica se daria então pelo tipo de dado trabalhado e ambas têm em comum o fato de privilegiarem dimensões espaciais em detrimento de outros visuais.

Para Manovich (2011, p. 38) essa diferenciação pode ser útil por permitir compreender as práticas de visualização da informação e design de informação sobrepostas parcialmente, mas, em última instância, diferentes, em termos de suas funções. O que é importante ressaltar, segundo Rodenbeck (2008, p.1), é que a visualização de informação tem se tornado mais do que um conjunto de ferramentas, tecnologias e técnicas, emergindo como um medium que possui uma vasta gama de possibilidades expressivas. Especificamente no caso das narrativas, o uso de múltiplos códigos semióticos em contar histórias envolve relações semióticas entre palavras, imagens e sons que podem enriquecer as oportunidades produtivas e perceptivas para contar histórias.

Conforme afirma Prince (2003, p. 1) não há um consenso sobre o que é exatamente narrativa. O autor salienta que alguns teóricos e pesquisadores acreditam que tudo é uma narrativa; outros afirmam que tudo pode ser; e outros ainda afirmam que, em certo sentido, nada é (porque é narratividade e vinculado ao contexto dependente da cultura). Entretanto, para fins metodológicos, seguiremos a conceituação do mesmo autor (Prince, 1982, p.4), segundo o qual, uma narrativa é "a representação de pelo menos dois eventos reais ou fictícios em uma sequência de tempo, nem de que pressupõe nem implica a outra". 
A opção em privilegiar essa definição que identifica a sequência temporal como a característica mais comum de narrativas e, na verdade, se deu, além do fato de que tal definição parece ser adotada por vários pesquisadores (Ochs e Capps 2001: 4), justamente porque essa definição não é limitada pela utilização de qualquer suporte particular, possibilitando uma abertura conceitual que nos permita abordar as narrativas nos contemporâneos ambientes de midiáticos. Ao enfatizar uma definição similar, enfatizando a dimensão crossmedia da narratividade, Meister, Kindt e Schernus (2005, p. xiv) afirmam que tal definição deve ser válida e significativa para todas as mídias que contêm representações e pode ser intuitivamente classificado como mídias possíveis de narrar. Dessa forma partiremos a seguir para a apresentação das características narrativas da obra "The Whale Hunt" de Jonathan Harris.

\section{Jonathan Harris e "The Whale Hunt":}

Jonathan Harris é um artista digital e cientista da computação. Harris estudou fotografia e ciência da computação na Universidade de Princeton (EUA) e seus projetos combinam elementos de ciência da computação, fotografia, arte visual e narrativa para compor uma poética dos dados. Em seu site, http://number27.org é possível encontrar uma compilação de sua obra, juntamente com uma breve descrição da ideia por trás de cada peça. Um de seus projetos mais interessantes é "Whale Hunt," de 2007, um trabalho precursor no uso criativo de banco de dados interativos.

Harris (2015, p.1) apresenta "Whale Hunt" como uma experiência narrativa. A proposta de Harris foi criar um experimento com uma série de fotografias tiradas durante a caça da baleia com arpão entre o povo esquimós inupiat em Barrow, no Alasca. Durante nove dias, 3.214 fotografias (figura 1) foram tiradas documentando o processo que se inicia com a proposta 
METAgraphias: letra F de \#foulipo (criação potencial) v.2 n.3 setembrol2017 0 design de informação e a narrativa diagramática em Whale Hunt de Jonathan Harris • Juliana Rocha Franco (judorf@gmail.com)

ainda no papel e termina com o abate de uma baleia sobre o gelo do Oceano Ártico.

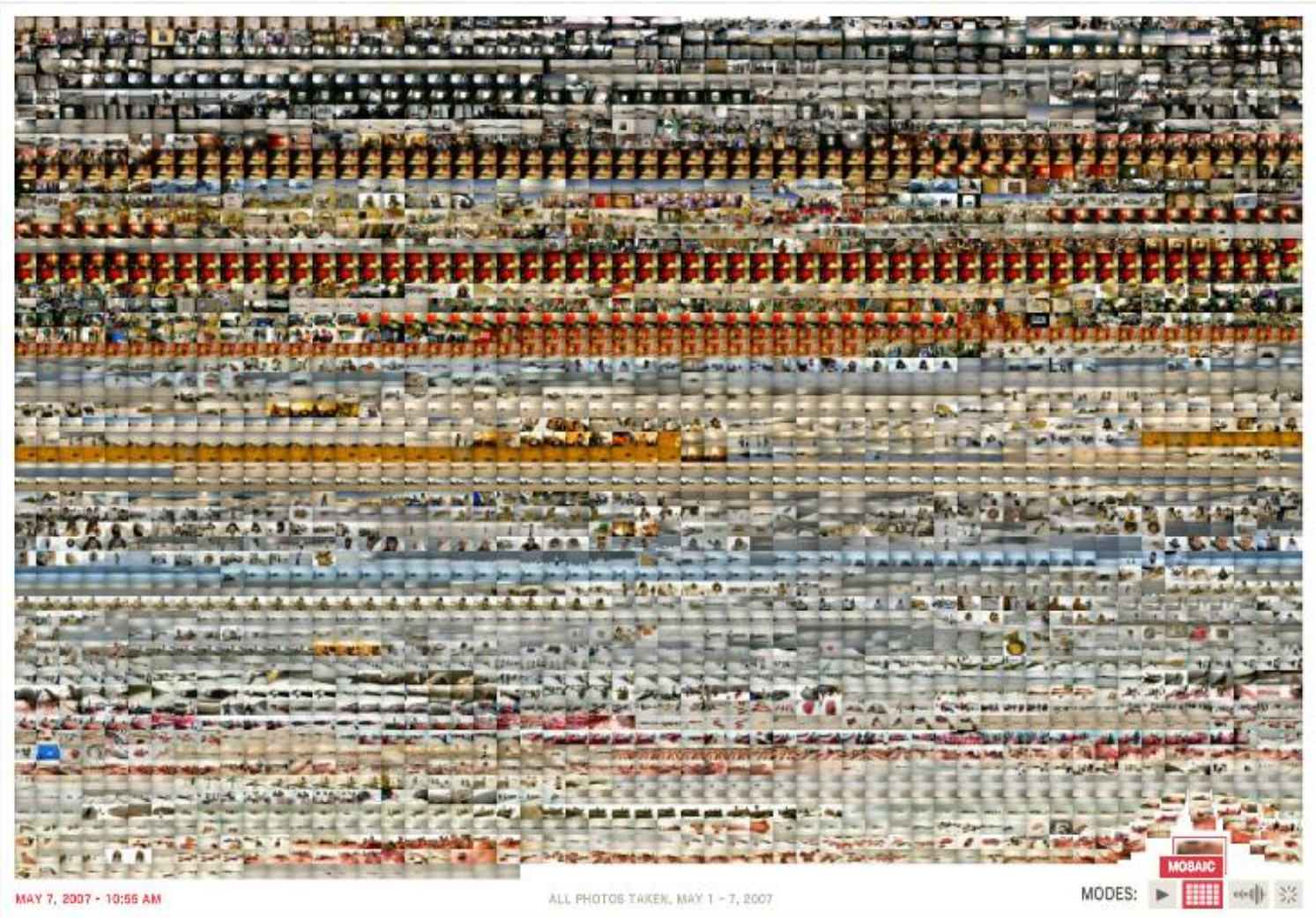

Figura 1 - HARRIS, Jonathan. Todas as fotos do projeto, Mosaic mode. Disponível em: < http://number27.org/whalehunt >. Acesso em: 2/2017.

Segundo Harris (2015, p.1), a caça de baleias Inupiat é uma tradição milenar que fornece o abastecimento alimentar anual da comunidade. Atualmente a caça é limitada a 22 baleias por ano. A cada primavera, com o degelo do oceano ocorre a quebra de uma parte do gelo agarrado ao continente que então flutua para fora ao mar, criando um canal de águas abertas chamado de "the lead". É através desses canais que baleias de Bowhead retornam da migração anual. É importante ressaltar que tal forma de caça de forma alguma se institui como ou se identifica com a caça comercial de baleias. Os aspectos da caça à baleia pelos esquimós se dá sob o controle dos rituais cerimoniais e sistemas de crença, bem como padrões habituais que perduram através de longeva tradição, fundamentais para a sobrevivência dessas 
comunidades ${ }^{1}$.

O artista documentou todo o processo, começando com a viagem de táxi para o aeroporto de Newark e terminando com o abate da segunda baleia, sete dias depois. As fotografias foram tiradas em intervalos de cinco minutos, mesmo enquanto o artista dormia, promovendo uma "pulsação fotográfica" constante. As fotografias foram tomadas pelo menos a cada cinco minutos em momentos de alta emoção e frequência das fotografias tiradas aumentava (taxa máxima de 37 fotos em cinco minutos). Os intervalos tempo representam o que Harris descreve como uma "pulsação fotográfica" em uma clara analogia com o batimento cardíaco e que depois ajudará a fornecer o ritmo para a narrativa.

Dado um registro com uma grande quantidade de dados, a questão que orientou Harris foi justamente em como essa narrativa poderia ser contada. O banco de dados não cria uma história para os quais são cruciais relações sequenciais de causa-efeito. Um banco de dados é uma variedade de escolhas, possibilidades; nesse caso o banco de dados pode ser compreendido como uma determinada ferramenta para a criação de narrativas. A partir do trabalho de fotografia documental, Harris constrói, um tratamento criativo de grandes quantidades de dados. O trabalho propõe uma nova interface para contar histórias, como veremos a seguir.

\section{A interface $e^{2}$ a narrativa ergódica como raciocínio diagramático:}

Como podemos observar na figura 2, na borda inferior da tela existe uma linha do tempo que

\footnotetext{
1 Para mais informações ver: BOCKSTOCE, John R. Whales, ice, and men: the history of whaling in the western Arctic. University of Washington Press, 1986.

${ }^{2}$ As informações descritivas da interface contidas no tópico abaixo são devedoras da própria descrição que Harris $(2015$, p.1) faz do trabalho.
} 
abarca a viagem inteira, começando com a corrida de táxi para o aeroporto de Newark e terminando com a caça da segunda baleia, sete dias depois. A linha é retratada de forma similar a um gráfico de batimento cardíaco médicos e cuja altura em cada ponto corresponde à frequência fotográfica. Harris afirma que a frequência das fotos está relacionada ao nível de excitação do momento fotografado. A posição da fotografia vista (no caso da figura 2, o amanhecer no acampamento) é indicada por uma barra vertical na linha do tempo. Ao mover o cursor do mouse ao longo da linha, as barras se espalham, facilitando para o usuário isolar um único momento no tempo. As 3.214 imagens são apresentadas na rolagem horizontal e para mover de uma fotografia para outra, existem, à esquerda e à direita da linha do tempo duas cabeças arpão, que funcionam como botões que acionam a mudança. 


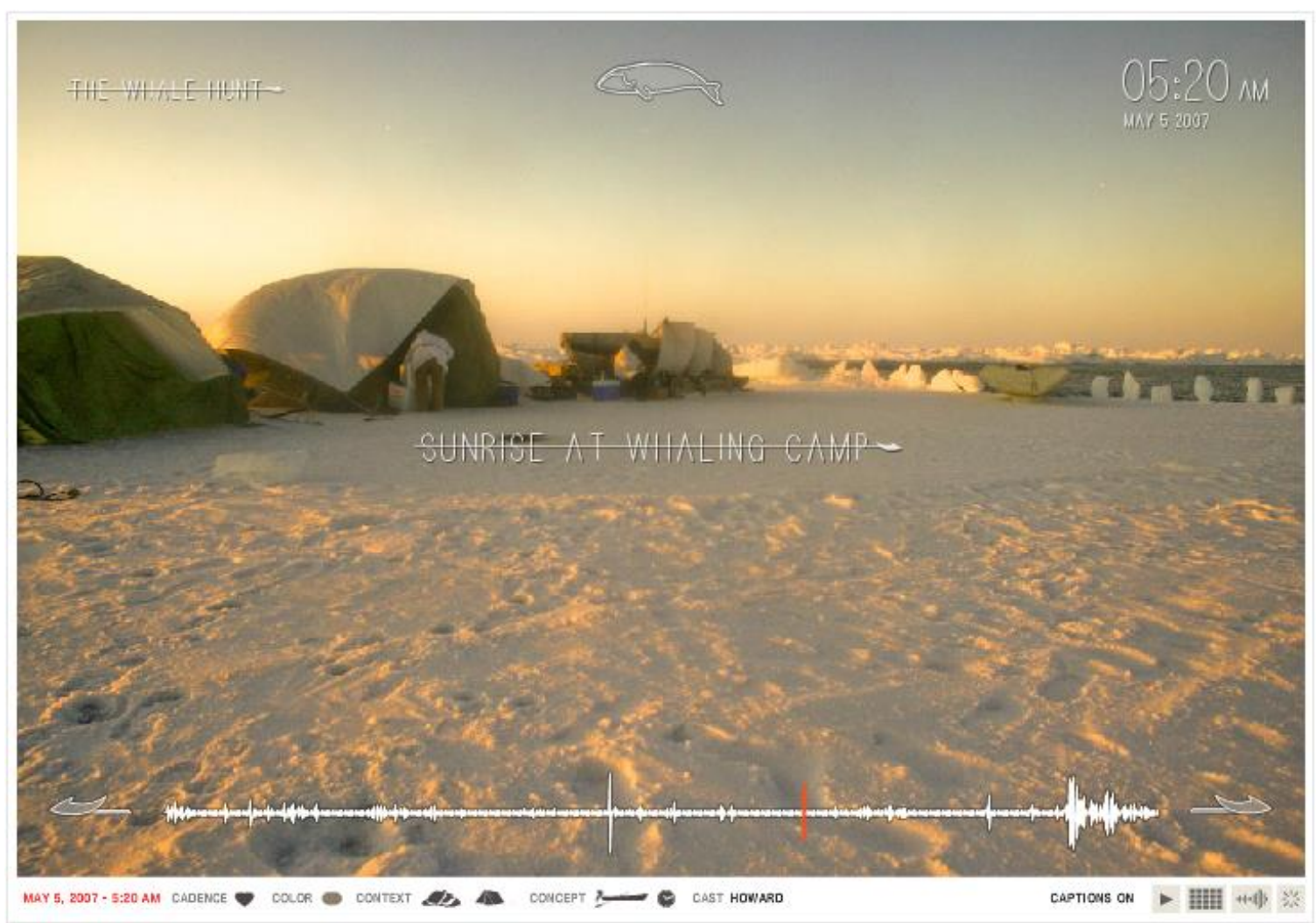

Figura 2 - HARRIS, Jonathan. Tela aleatória do Projeto. Disponível em: < http://number27.org/whalehunt >. Acesso em: 2/2017

Observa-se no canto inferior da tela um botão que alterna a preferência entre fotos com legenda ou sem legendas, que fornecem um breve comentário sobre a fotografia que está visível. Na barra abaixo da timeline é possível ver pequenos textos e ícones, indicando uma grande variedade de metadados sobre a fotografia que está visível, como por exemplo, a cor predominante em cada fotografia, calculada pela cor média dos pixels de cada uma, a data e hora a foto foi tirada, dentre outros.

Harris (2015, p.1) elaborou o que ele chama de "restrições" ou filtros (figura 3), que possibilitam navegar pelas imagens a parir de determinadas escolhas. Centrada no topo da tela existe o ícone de uma baleia ártica. Clicando nesse ícone abre-se o painel de restrições, que são ferramentas que podem ser usadas para elaborar e isolar várias narrativas. Ao aplicar 
as restrições a linha do tempo modifica-se a forma que os dados podem ser apresentados. As restrições listadas são a "Cadencia" que destaca o qual era o nível de excitação naquele momento. $O$ "Contexto" que relaciona a foto ao local que ela foi tirada (por exemplo, New York City, aviões e aeroportos; Barrow, no Alasca, a casa da família Patkotak, o Oceano Ártico, o campo de caça à baleia Patkotak, acampamento, baleeira etc.)

Outra restrição se dá por "Conceitos", que são vários elementos e ideias que as ideias estão apresentados na foto (como por exemplo sangue, barcos, edifícios, alimentos, jogos, crianças, Moby Dick, etc.), e por fim, a restrição "Elenco", que separa por personagens envolvidos na história (Abe, Ahmakak, Andrew, Crawford, David, Eduardo, Howard, Joe, Jonathan Josias, Kat, Ransom, Rony, Rony, Samuel, Simeão, Walter, o 1 baleia, a baleia e 2). 

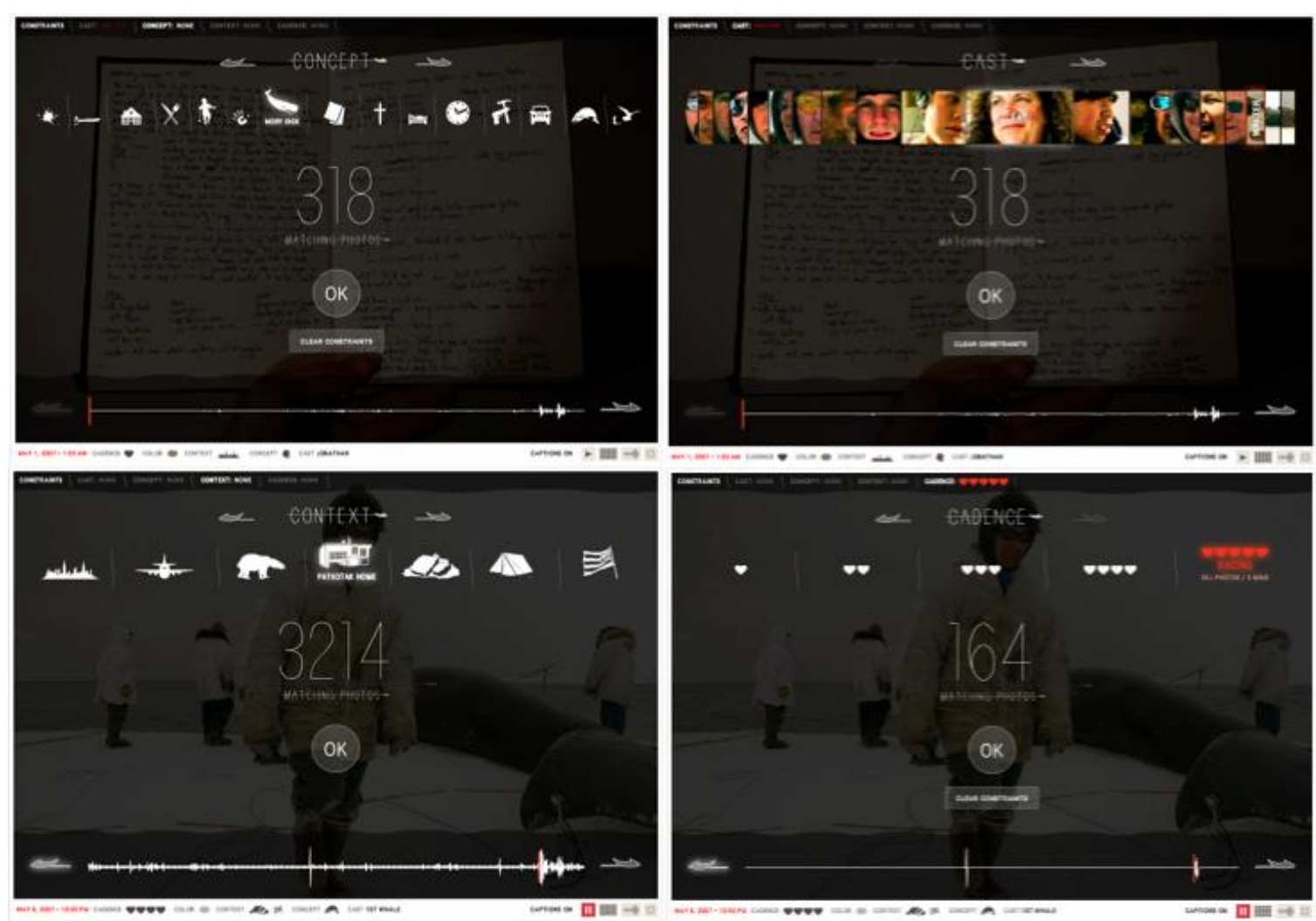

Figura 3 - HARRIS, Jonathan. Quatro telas de restrições. Disponível em: < http://number27.org/whalehunt $>$. Acesso em: 12/2015

Ao propor uma narrativa não-linear e comutável, Harris torna a interação do usuário/espectador fundamental e cria o que Aarseth (2006) denomina ser um cibertexto. O conceito de cibertexto (AARSETH, 2006, p. 19) centra-se na organização mecânica do texto, reconhecendo as implicações do médium como parte integrante do intercâmbio literário. Contudo, também centra a sua atenção no consumidor ou utilizador do texto. Segundo o autor (2006, p.19), o desempenho do seu leitor realiza-se todo no seu cérebro, enquanto que o do utilizador do cibertexto também se exerce num sentido extranoema?tico.

O cibertexto originaria o que o autor denomina narrativa ergo?dica. Aarseth toma emprestado o termo ergodic que deriva das palavras gregas ergon e hodos, que significam "obra" e "via" 
(AARSETH, 2006, p. 19). O autor define narrativa ergódicas como aqueles que exigem "esforço não trivial" do leitor que percorre o texto. Dessa forma, independente do suporte no qual esteja inscrita, a narrativa ergo? dica seria aquela que exige a participação do leitor (AARSETH, 2006, p. 115).

Na literatura ergódica, um "esforço relevante" é requerido por parte do leitor para passar pelo texto. Se a literatura ergódica faz sentido como um conceito válido, então também deve haver uma literatura não ergódica, onde o esforço para percorrer o texto é trivial, sem responsabilidade extrano-estatística para o leitor, além de mover os olhos ou virar as páginas.

Harris efetivamente apresenta um processo de animação de uma grande quantidade dados, organizados em narrativas hipertextuais de forma bastante acessíveis, funcionais, intuitivas e esteticamente atraentes. Um conjunto de escolhas que alteram a configuração do texto. Segundo Aarseth (2006. P.19) o usuário efetua uma sequência semiótica através de um movimento de seleção. É justamente esse que o autor denomina ergódico: "podemos definir um texto ergódico como aquele em que está presente, além da função interpretativa obrigatória, pelo menos uma das quatro funções do utilizador." (AARSETH, 2006, p. 86). Em Whale Hunt o usuário não só interpretá a narrativa, como também a explora e a configura. A premissa básica é a da agência como interação (AARSETH, 2006, p. 2).

É importante ressaltar que para Aarseth, existem três tipos de configurações discurso: 0 ergódico, o narrativo e o descritivo. Eskelinen $(2012$, p. 88) afirma que tais discursos podem coexistir em níveis: "We already know that the ergodic side can coexist and be combined with traditional text types (argument, description and narrative)". Dessa forma, embora a questão ergódica seja a proeminente, é possível vislumbrar elementos que sugerem a presença dos outros discursos, como por exemplo como elementos descritivos (legendas e textos 
indicativos), mas que precisam ser escolhidos e/ou combinados ergoicamente pelo usuário.

O princípio subjacente de uma narrativa a partir de um banco de dados seria uma poética recombinante. Aqui, como em anteriores ensaios, combinação, categorização, indexação são reconhecidos como princípios essenciais de banco de dados que têm um impacto profundo sobre a narrativa. Várias restrições podem ser combinadas para criar narrativas. Por exemplo, pode-se isolar a história de "sangue e veículos no Oceano Ártico, envolvendo Simeão e Crawford".

Dessa forma é possível afirmar que a estrutura narrativa funciona como manobra semiótica diagramática na medida em que se constitui de "textos abertos e dinâmicos onde o leitor deve realizar ações específicas para gerar uma sequência literária, que pode variar para cada leitura" (MURRAY, 2006; 4). O processo do raciocínio diagramático implica a formação na mente de algum tipo de representação icônica dos fatos, aqui especificamente, os dados. Ao se observar uma imagem visual, a partir de um banco de dados, "The Whale Hunt" permite conceber a presença de relações que abstratamente expressas por elementos formais imaginados de acordo com certas regras. A narrativa se estabelece na possibilidade de relação entre as partes. Funciona permitindo mapear e experienciar sobre possibilidades oferecidas pelo banco de dados. Nesse caso o contexto narrativo se estrutura como base para cognição e comunicação quando a narrativa se estabelece como como processo diagramático.

\section{Considerações finais}

O Design de informação se configura como reto?rica crucial para semioses coletivas e se configura como um conjunto retórico que permite compartilhar conhecimentos sobre 0 mundo a partir de um vasto conjunto de dados. 
Padrões de cultura normalmente manifestam-se em uma variedade de materiais culturais. $\mathrm{O}$ trabalho de Harris é de 2007, e o que se percebe cada vez mais, é um processo de consolidação de várias sintaxes para a visualização de informação que na prática, trazem preceitos, protocolos e prescrições que conduzem o arranjo para uma prática de interpretação com alguma aproximação para o que Neves denomina classes de expressão pública. Conforme afirma Neves (2010, p.7), "máquinas lógicas de percepção carregam muitas determinações próprias ausentes da percepção desaparelhada (recortes espaço- temporais, limiares de sensibilidade), são reincorporadas aos hábitos dos públicos seus contemporâneos, assimiladas aos processos perceptivos preexistentes".

No caso de "Whale Hunt" podemos argumentar, via Neves (2010) que diversos procedimentos de criação de estruturas de visualização de informação se explicam através da "comunicação simbólica, por meio da qual os públicos intérpretes compartilham certos "protótipos argumentativos. Cada enunciação "replica" - ou seja, atualiza e transforma de maneira mais ou menos explícita — os princípios e parâmetros gerais. Isso explica talvez a enorme recorrência das representações estruturadas em linha do tempo, em diversas representações diagramáticas contemporâneas, inclusive em Jonathan Harris.

Isso possibilita um processo de simbolização de estratégias na construção de artefatos argumentativo-narrativos. Porém, ao preço de ter reduzido tais enunciações proposições não articuladas em argumentos. Desta forma é possível afirmar que o futuro do design de informação ainda possui o desafio poético-político da invenção de mecanismos de reflexivização e performatização dos atos de apreciação de arquivos para além das repetições e recorrências argumentativas. Porque conforme afirma Hoffman (2004, p. 301), não é suficiente, que nós executemos nossas experiências com diagramas quase às cegas, como 
uma máquina. Apesar de um computador pode realizar experiências muito melhores do que os seres humanos, o ponto essencial da realização dos limites de um sistema de representação selecionado é a autorreflexão na qual a criatividade do raciocínio diagramático é realizada principalmente na modificação dos sistemas de representação, na adição de novos meios ou na reconstrução de sua ordem sistemática.

\section{Referencias Bibliográficas:}

AARSETH, A. J. Cibertext: perspectives on ergodic literature. London: The Johns Hopkins University Press, 1997. BOCKSTOCE, John R. Whales, ice, and men: the history of whaling in the western Arctic. University of Washington Press, 1986.

BORDWELL, David. Narration in the fiction film. Routledge, 2013.

BORDWELL, David. ApProppriations and imPropprieties: Problems in the morphology of film narrative. Cinema journal, p. 5-20, 1988.

CHEN, C. Information Visualization Beyond the Horizon. London: Springer London, 2006.

ESKELINEN, Markku. Cybertext poetics: the critical landscape of new media literary theory. Bloomsbury Publishing USA, 2012.

GUIMARAES, Celio Cardoso. Fundamentos de bancos de dados: modelagem, projeto de linguagem SQL. Ed. da Unicamp, 2003.

HARRIS, Jonathan. The Whale Hunt. Disponível em: < http://number27.org/whalehunt >. Acesso em: 12/2015.

HOFFMANN, Christian R. (Ed.). Narrative revisited: telling a story in the age of new media. John Benjamins Publishing, 2010.

HOU, J. L.; PAI, S. T. A spatial knowledge sharing platform. Using the visualization approach. International Journal of Production Research, v. 47, n. 1, p. 25-50, 2009.

MANOVICH, L. What is visualization? Visual Studies, v. 26, n. 1, p. 36-49, 2011.

MEIRELLES, I. Information design resource prototype: an introduction. 2005. Disponível em: < http://nuweb.neu.edu/mmeirelles/infoDesignResource/ >. Acesso em: 7/2014.

. Diagrams and problem solving. 2007. Brazilian Society of Information Design, 2007. p.45-53. 
METAgraphias: letra F de \#foulipo (criação potencial) v.2 n.3 setembrol2017 0 design de informação e a narrativa diagramática em Whale Hunt de Jonathan Harris • Juliana Rocha Franco (judorf@gmail.com)

Design for information: an introduction to the histories, theories, and best practices behind effective information visualizations. Beverly: Rockport, 2013.

MEISTER, Jan Christoph; KINDT, Tom; SCHERNUS, Wilhelm. Narratology beyond literary criticism: Mediality, disciplinarity. Walter de Gruyter, 2005.

MEISTER, Jan Christoph. Computing action: a narratological approach. Walter de Gruyter, 2003.

NADEL, Alan. Second Nature, Cinematic Narrative, the Historical Subject, and Russian Ark. A Companion to Narrative Theory, p. 427-440, 2005.

NEVES, Bráulio de B. Imagens-câmera, máquinas lógicas e retóricas documentárias. Revista Semeiosis-semiótica e transdisciplinaridade em revista, no, 2010.

OCHS, Elinor; CAPPS, Lisa. A dimensional approach to narrative. Living narrative: Creating lives in everyday storytelling, 2001.

PAUL, Christiane. The Database as System and Cultural Form: Anatomies of Cultural Narratives. Disponi?vel em: <www.cityarts.com/paulc/RISD/Paul_Database.doc>. Acesso em: 12/2015

PRINCE, Gerald. Narratology: The form and functioning of narrative. Walter de Gruyter, 1982.

QUAGGIOTTO, M. A new atlas for abstract spaces. Visual tools for the exploration of complex contexts. Design Research Society (DRS) international conference Design \& Complexity Montreal (Quebec), Canada, 2010.

RODENBECK, Eric. Information Visualization is a Medium. In: Keynote lecture delivered at Emerging Technology Conference, San Diego, California, March. 2008. p. 3-6.

SCHIFFER, Sheldon. The Rise and Fall of Black Velvet Flag An "intelligent" system for youth. In: MATEAS, Michael; SENGERS, Phoebe (Ed.). Narrative intelligence. John Benjamins Publishing, 2003.

TERGAN, S. Knowledge and information visualization searching for synergies. Berlin; New York: Springer, 2005.

VAN DER VLIST, Fernando N. Distributed Narratives Extracting Design Methods from Nonlinear Narrative Formations. 2012. Disponi? $\mathrm{vel}$ em: < http://fernandovandervlist.nl/papers/distributed-narratives.html>. Acesso em: $12 / 2015$

VESNA, V. Database aesthetics. Art in the Age of Information Overflow. Minneapolis: University of Minnesota Press, 2007.

WRIGHT, S. Dataesthetics reader how to do things with data. Frankfurt: Revolver, Archiv für aktuelle Kunst, 2007. 